\title{
Activated Wnt signaling promotes growth and progression of AFP-producing gastric cancer in preclinical models
}

This article was published in the following Dove Medical Press journal: Cancer Management and Research

\author{
Dongshao Chen ${ }^{1, *}$ \\ Xiaoting $\operatorname{Lin}^{1, *}$ \\ Cheng Zhang' \\ Guo $A n^{2}$ \\ Zhongwu $\mathrm{Li}^{3}$ \\ Bin Dong ${ }^{3}$ \\ Lin Shen' \\ Jing Gao' \\ Xiaotian Zhang' \\ 'Department of Gastrointestinal \\ Oncology; ${ }^{2}$ Department of Laboratory \\ Animal; ${ }^{3}$ Department of Pathology, \\ Key Laboratory of Carcinogenesis and \\ Translational Research (Ministry of \\ Education, Beijing), Peking University \\ Cancer Hospital and Institute, Beijing \\ I00I42, China
}

*These authors contributed equally to this work

Correspondence: Xiaotian Zhang; Jing Gao

52 Fu-Cheng Road, Haidian, Beijing 100142, China

Tel $+861088196561 ;+861088196747$

$\mathrm{Fax}+861088196561$

Email zhangxiaotianmed@I63.com; gaojing_pumc@163.com
Background: Characterized by elevated AFP levels in serum, AFP-producing gastric cancer (APGC) is a very special type of gastric cancer (GC) that is difficult to treat and has poor prognosis. However, little is known about the role of AFP in GC, which was investigated in this study with in vitro and in vivo experiments.

Methods: APGC cells were established with lentivirus infection and validated by PCR assay and ELISA in HCG27 and AGS cells. Cell growth, migration, and invasion were determined by CCK8, transwell assays, and animal experiments. RNA sequencing, Western blot, dualluciferase-reporter assays, and RNA interference were employed to understand mechanisms underlying AFP activity, followed by therapeutic investigations for APGC.

Results: APGC cells featured significantly increased AFP levels in cellular supernatants. AFP potentiated growth and aggression in GC cell lines and their derived xenografts. Wnt-signaling activation was responsible for AFP function, indicated by decreased Axin 1 and pGSK3 $\beta$, followed by cascade activation of $\beta$-catenin, downstream transcription factors TCF1/TCF7, and the target gene $-c-M y c$. Wnt-signaling blockade by Axin 1 rescue or pathway inhibitor XAV939 reversed AFP function, suggesting the potential therapeutic value of APGC.

Conclusion: AFP played a critical role in APGC through activating Wnt signaling, and targeting Wnt pathways might be a promising strategy against APGC.

Keywords: alpha-fetoprotein, AFP, AFP-producing gastric cancer, APGC, Axin 1, Wnt signaling, Wnt-signaling inhibitor

\section{Introduction}

Despite the fact that the management of gastric cancer (GC) has progressed greatly, its high metastasis indicates few chances for curative surgery and remains a leading cause of therapeutic failure and cancer-related mortality. ${ }^{1}$ Therefore, uncovering the molecular mechanisms of cancer progression and developing corresponding targeted therapies are crucial to improve GC outcomes.

AFP-producing gastric cancer (APGC), a most aggressive subtype of GC, has recently attracted much attention. Initially reported in 1970, APGC is characterized by a high incidence of venous invasion, lymphatic invasion, and liver metastasis compared with AFP-negative GC. ${ }^{2}$ Due to its high malignancy, APGC displays the poorest overall prognosis and urgently demands more effective treatment strategies. ${ }^{2,3}$ However, routine treatment, including radical resection and chemotherapy regimens, achieves limited therapeutic effectiveness against APGC. ${ }^{2,4}$ Optimal management for APGC, especially 
liver metastasis, which serves as an independent prognostic factor, ${ }^{3-6}$ is of pressing need to be developed.

High expression or elevated serum levels of AFP have been reported to correlate positively with cancer aggression, represented by liver metastasis. ${ }^{3-6}$ Preliminary studies have demonstrated enhanced growth, apoptosis, angiogenesis, metastasis, and cancer stem cell (CSC)-like properties in AFP-producing hepatocellular carcinoma (HCC), ${ }^{7-11}$ conferred by PI3K-Akt signaling. ${ }^{7-9}$ With regard to GC, few data have sporadically revealed AFP-promoted in vitro invasion and in vivo growth. ${ }^{12,13}$ Genomic or proteomic analyses identify overexpression of CSC-like markers, angiogenic factors, and antiapoptotic proteins in AFPproducing tumors compared to conventional cancers. ${ }^{14-16}$ Of interest, high expression of CSC or angiogenic markers, including SALL4, VEGF, and c-Met, have been detected by immunohistochemistry (IHC) in APGC tissues and cell lines compared to AFP-negative GC. ${ }^{17-21}$ As such, pathways involving CSCs or angiogenesis may be critical events and potential targets for AFP-producing cancers. Taken together, AFP-mediated malignant phenotypes, especially in secreted form, remain poorly understood, and excavating molecular mechanisms (PI3K-Akt, CSC, and angiogenic pathways, in particular) are pivotal to designing rational strategies and gaining survival benefits for patients with APGC. In this study, we comprehensively investigated AFP's roles in aggressive behaviors and its mechanisms using GC preclinical models accompanied by therapeutic potentials of targeting the pathways for APGC. Our work sheds light on novel therapeutic options for APGC and provides rationale for future clinical studies.

\section{Methods}

\section{Reagents and antibodies}

XAV939 was purchased from Selleck Chemicals (Houston, TX, USA). The reagent was formulated and stored following the manufacturer's protocol. Primary antibodies against $\beta$-catenin (8480), active $\beta$-catenin (8814), Axin 1 (2074), GSK3 $\beta$ (12456), TCF1/TCF7 (2203), c-Myc (5605), AFP (3903), and secondary HRP-conjugated goat antirabbit (7074) and antimouse antibodies (7076) were purchased from Cell Signaling Technology (Danvers, MA, USA). The pGSK3 $\beta Y 216$ (ab75745) antibody was purchased from Abcam (Cambridge, UK). Antibodies against $\beta$-actin (A5441) and Ki67 (ZM0167) were purchased from SigmaAldrich Co. (St Louis, MO, USA) and ZSGB-Bio (Beijing, China), respectively.

\section{Cell lines and cell culture}

The human AGS GC cell line was purchased from the American Type Culture Collection (Manassas, VA, USA) and kindly provided by Professor Youyong Lu (Peking University Cancer Hospital and Institute). ${ }^{22,23}$ Human HGC27 GC cell line and liver cancer cell line HepG2 were purchased from Bank of Chinese Academy of Sciences (Beijing, China). Cell lines were incubated at $37^{\circ} \mathrm{C}$ in a humidified atmosphere with $5 \%$ $\mathrm{CO}_{2}$ and cultured in RPMI 1640 medium (Thermo Fisher Scientific, Waltham, MA, USA) supplemented with 10\% FBS (Thermo Fisher Scientific) and 1\% penicillin-streptomycin (HyClone, Logan, UT, USA).

\section{Genetic overexpression and knockdown}

Lentiviral constructs expressing AFP and control vector purchased from GeneCopoeia (Rockville, MD, USA) were transfected into GC cells for 48 hours. Infected cells were cultured in selective medium containing $2 \mu \mathrm{g} / \mathrm{mL}$ puromycin (InvivoGen, San Diego, CA, USA) for 1-2 weeks to establish stably-infected cell lines used in subsequent experiments.

Cells were seeded in six-well plates and transfected at about $80 \%$ confluence by Lipofectamine 3000 (Thermo Fisher Scientific) according to the manufacturer's instructions. HepG2 cells were transfected with AFP siRNAs and their negative control siRNA using an AFP-siRNA kit (RiboBio, Guangzhou, China), GC cells with Axin 1 siRNAs, and their negative control siRNA using an Axin 1-siRNA kit (RiboBio) and AFP-overexpressing GC cells with Axin 1 and its control plasmids (Vigene Biosciences, Jinan, China). Cells transfected for 72 hours were harvested for immunoblotting analysis.

\section{RNA extraction and reverse- transcription (RT)-PCR}

Cellular total RNA was extracted with Trizol reagent (Thermo Fisher Scientific) and cDNA templates were generated via RT-PCR using high-capacity RNA-to-cDNA kits (Applied Biosystems, Carlsbad, CA, USA). PCR was performed with $2 \mu \mathrm{L}$ synthesized cDNA using an LA PCR Kit (Takara Bio, Kusatsu, Japan), and the housekeeping gene $G A P D H$ was employed as the internal control. PCR products were detected by $1 \%$ agarose-gel electrophoresis. The primers of AFP and GAPDH were: AFP forward, 5'-TCAGTGAGGACAAACTATTGG-3'; AFP reverse, 5'-CTCTTCAGCAAAGCAGACTTC-3'; GAPDH forward, 5'-GCACCACCAACTGCTTAGC-3'; and GAPDH reverse, 5'-GGCATGGACTGTGGTCATA-3'. 


\section{ELISA analysis}

A human AFP ELISA kit (ab193765) was purchased from Abcam. The ELISA plate was coated with AFP-capture antibody able to conjugate AFP in cell-culture supernatants. In accordance with the vendor's instructions, supernatants of AFP-overexpressing and control GC cells with a serial dilution of standards were added to respective wells, followed by antibody cocktails. The plate was sealed and incubated with shaking for 1 hour at room temperature. After being washed, the plate was incubated with $100 \mu \mathrm{L}$ tetramethyl benzidine substrate for 10 minutes in the dark and $100 \mu \mathrm{L}$ Stop solution for 1 minute on a plate shaker. Intensity was measured at 450 $\mathrm{nm}$ using spectrophotometry. According to standard curves, test supernatant concentrations were calculated.

\section{Cell-viability assays}

Cells $(5,000 /$ well) were seeded into 96-well plates and allowed to adhere overnight in complete medium. After treatment, cell viability was measured using a CCK8 kit (Dojindo Laboratories, Tokyo, Japan) according to the manufacturer's protocol. Absorbance was measured at 450 $\mathrm{nm}$ using spectrophotometry.

\section{Cell-invasion and -migration assays}

For invasion and migration assays, cells suspended in serumfree medium were added into the upper chambers of 24-well transwell plates with/without precoated Matrigel (Corning, New York, NY, USA), respectively. Lower chambers were filled with culture medium supplemented with $10 \%$ FBS. Invaded and migrated cells in lower chambers were fixed and stained with crystal violet and counted under microscopy after 36 and 24 hours' incubation, respectively.

\section{Luciferase-reporter gene assays}

TOPflash/FOPflash (TCF wild-type/mutated control) luciferase reporter plasmids and Renilla plasmids were purchased from FenghBio (Changsha, China). TOPflash and FOPflash plasmids (500 ng) were separately cotransfected with $25 \mathrm{ng}$ Renilla plasmid into cells seeded in 24-well plates using Lipofectamine 3000 (Thermo Fisher Scientific). After 48 hours' transfection, luciferase activity was measured with a dual-luciferase reporter assay (Promega Corporation, Madison, WI, USA) and normalized to Renilla. $\beta$-catenin-mediated TCF transcriptional activity was assessed by TOPflash:FOPflash ratios.

\section{RNA sequencing}

We prepared total RNA using the Trizol method and confirmed RNA integrity using a 2100 Bioanalyzer (Agilent Technologies,
Santa Clara, CA, USA). We performed next-generation sequencing using a HiSeq system according to the manufacturer's instructions (Illumina, San Diego, CA, USA). Sequences were processed and analyzed by Novogene (Beijing, China).

\section{Immunoblotting analyses}

GC cells and tumor tissue were lysed using a CytoBuster protein-extraction reagent (Merck Millipore, Darmstadt, Germany) in the presence of protease and phosphataseinhibitor cocktail tablets (Roche, Basel, Switzerland). Protein concentrations were measured with a BCA protein-assay Kit (Beyotime, Haimen, China). Soluble lysates were subjected to SDS-PAGE and transferred to polyvinylidene difluoride membranes (Merck Millipore). After 10 minutes' washing in TBST buffer three times, membranes blocked with 5\% BSA (Amresco, Solon, OH, USA) were probed with primary antibodies at $4{ }^{\circ} \mathrm{C}$ overnight and secondary antibodies $(1: 2,000)$ at room temperature for 1 hour. Signals were visualized using an Amersham Imager 600 (GE Healthcare, Little Chalfont, UK) after incubation with Clarity Western ECL substrate (Bio-Rad Laboratories Inc., Hercules, CA, USA). $\beta$-actin was used as the loading control.

\section{Animal experiments}

With three methods (subcutaneous, intraperitoneal, and caudal vein injections to assess tumorigenic, local dissemination, and distant metastasis capacity, respectively), PBS-suspended AFP-overexpressing HGC27 cells and controls were inoculated into 5-week-old female nonobese diabetic/severe combined immunodeficiency mice (five mice/group; Vital River Laboratories, Beijing, China). For tumorigenic experiments, tumor volume was measured every 3 days and calculated by the formula $\mathrm{V}=\mathrm{L} \times \mathrm{W}^{2} \times 0.5(\mathrm{~V}$, volume; $\mathrm{L}$, length; $\mathrm{W}$, width of tumor). After the final observation, mice were sacrificed by cervical dislocation and tumor tissue stripped and prepared for IHC and immunoblotting analyses. For metastasis experiments, peritoneal spreading and lung metastasis were assessed by necropsy 10 weeks after injection. Lungs, livers, and spleens were excised and embedded in paraffin for hematoxylin and eosin staining. All animal experiments followed laboratory animal guidelines of Beijing municipality for ethical review of animal welfare (http://www.baola.org) and were approved by Peking University Cancer Hospital's Institutional Animal Care and Use Committee.

\section{Immunohistochemistry}

After dewaxing, hydration, endogenous peroxidase removal, and blocking with $5 \%$ BSA according to standard procedures, 
$4 \mu \mathrm{m}$-thick formalin-fixed, paraffin-embedded sections were incubated with the primary antibody against Ki67 (1:300) overnight at $4^{\circ} \mathrm{C}$, followed by IgG-HRP polymer (ZSGBBio) and diaminobenzidine substrate (Gene Tech, Shanghai, China) in accordance with protocols. Two pathologists from the Department of Pathology at Peking University Cancer Hospital and Institute independently evaluated staining results as described in our previous study. ${ }^{24}$

\section{Statistical analysis}

All data are representative of three independent experiments and expressed as mean \pm SD. Differences between groups were analyzed by one-way or repeated-measure ANOVA using SPSS version 20.0 software (IBM Corporation, Armonk, NY, USA), and $P<0.05$ was considered statistically significant.

A
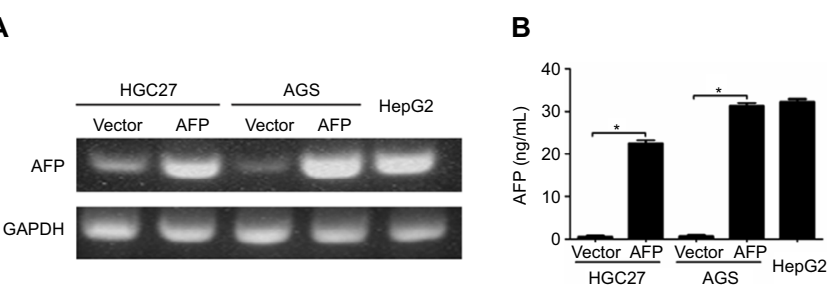

D
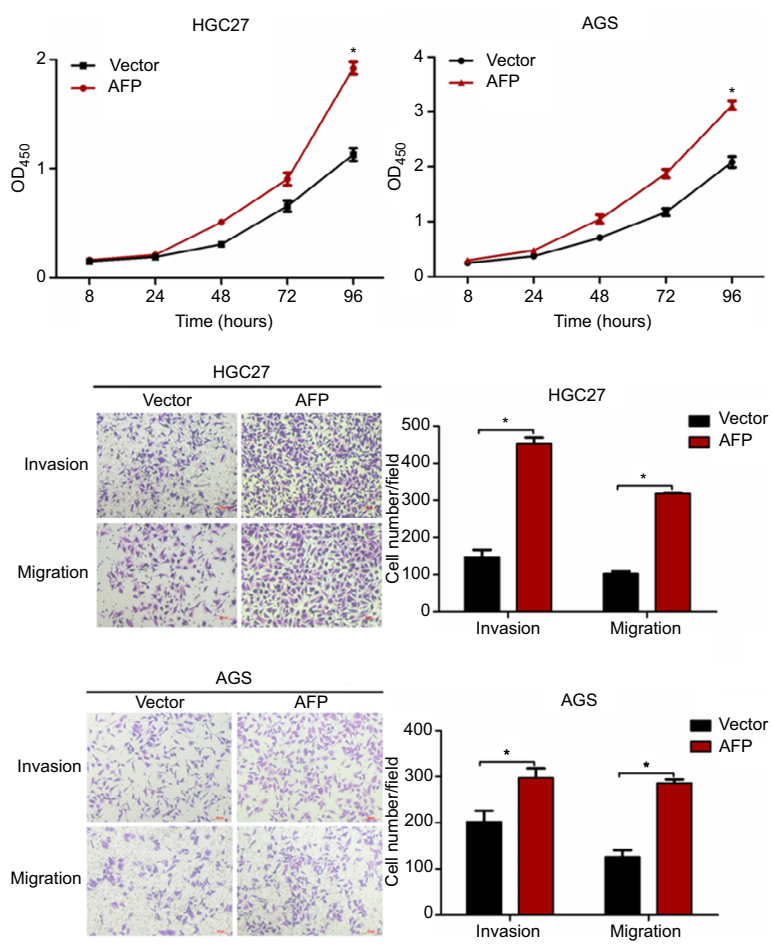

\section{Results}

\section{AFP overexpression and derived} supernatant enhanced proliferation, invasion, and migration in established APGC cells

Elevated AFP in serum or tissue has been identified to be associated with poorer prognosis in APGC than AFPnegative GC. ${ }^{3-6}$ To determine AFP's role in malignant GC phenotypes, we established AFP-overexpressing cell sublines by lentivirus infection with two selected AFP-negative GC cell lines (HGC27 and AGS). The two GC-cell sublines displayed enhanced AFP mRNA expression and secretion capability over their counterparts, which were maintained for a long period (Figure $1 \mathrm{~A}-\mathrm{C}$ ), indicating APGC preclinical models had been established. Of interest, proliferation, invasion, and migration abilities in AFP-overexpressing

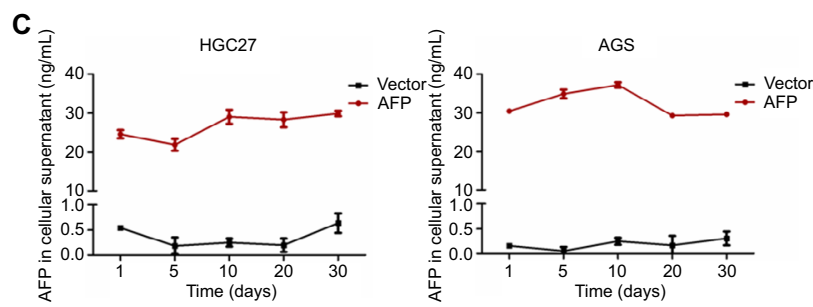

E
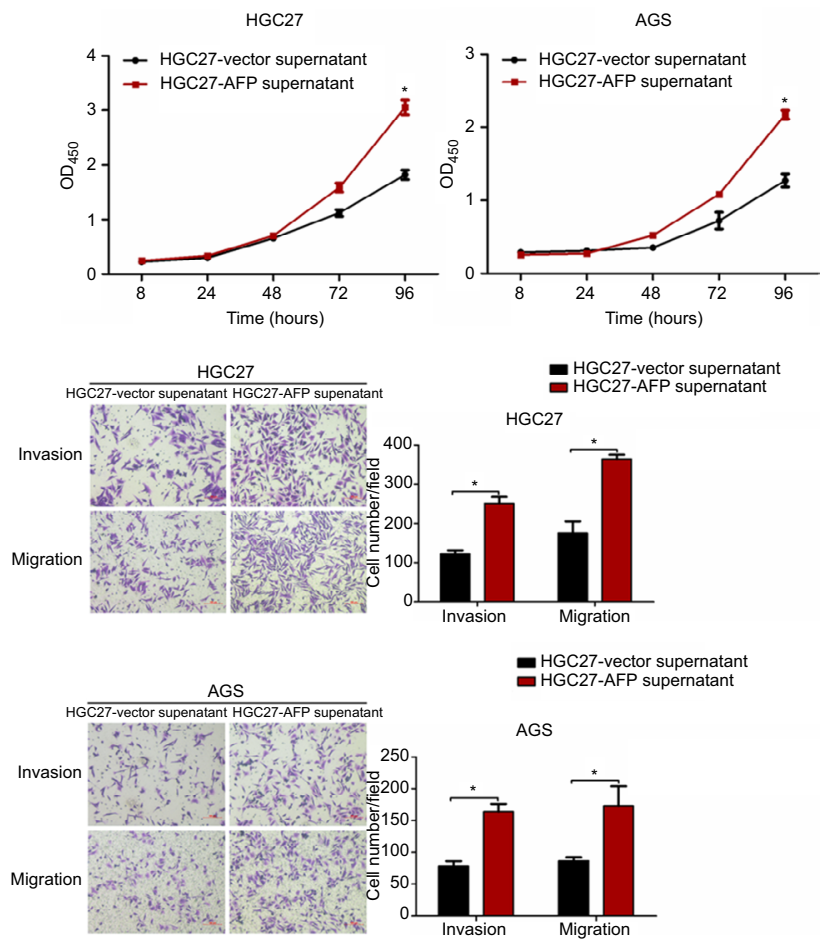

Figure I AFP overexpression and derived supernatant enhanced proliferation, invasion, and migration in established APGC cells.

Notes: (A and B) AFP overexpression in GC cells (HGC27 and AGS) was confirmed by PCR and ELISA with HepG2 cells as the positive control. (C) AFP-secretion levels of AFP-overexpressing GC cells compared to their controls were dynamically monitored by ELISA. (D and E) Impacts of AFP overexpression and derived supernatant on cell proliferation, invasion, and migration abilities in GC cells were measured by CCK8 and transwell assays, respectively. $* P<0.05$ by $A N O V A$. Data expressed as mean \pm SD. Abbreviation: APGC, AFP-producing gastric cancer. 
groups were superior to their controls (Figure 1D). To define further whether these AFP-mediated GC biological functions stemmed from its abnormal secretion, supernatants from HGC27-AFP or HGC27-vector cells were used as the stimulator. As expected, AFP supernatants yielded similar effects on GC proliferation, invasion, and migration as AFP overexpression (Figure 1E). Therefore, APGC cells promoted growth and progression via secretion, consistent with the poor prognostic value of elevated AFP in APGC serum and tissue.

\section{AFP promoted growth and metastasis in established APGC xenografts}

Based on AFP-mediated growth and progression observed in vitro, mice xenografts bearing HGC27-AFP and HGC27vector cells were used for in vivo assessment. Compared to the control, AFP overexpression significantly accelerated in vivo GC-tumor growth, further validated by a higher proliferation rate marked by enhanced Ki67 immunostaining (Figure 2A and B). In parallel with in vitro findings, mice injected intraperitoneally with AFP-overexpressing HGC27 cells were more prone to ascites and peritoneal dissemination (for HGC27-AFP and HGC27-vector groups, 1 [20\%] vs 0 and 4 [80\%] vs 0 mice developed ascites and peritoneal spreading, respectively; Figure 2C, E, and F). Moreover, lung metastasis occurred in one of five mice injected in the caudal vein with AFP-overexpressing HGC27 cells, whereas none was observed in the control group (Figure 2D and F). These data suggested AFP-facilitated local dissemination and distant metastasis of GC in vivo. Taken together, AFP promoted GC growth and metastasis in vivo.

\section{AFP activated canonical Wnt signaling in established APGC preclinical models}

To unmask the potential molecular mechanisms underlying AFP-promoted GC malignancy, RNA-sequencing analyses were performed in HGC27-AFP and HGC27-vector cells. Kyoto Encyclopedia of Genes and Genomes pathway analyses unveiled Wnt signaling as the top enriched candidate pathway (Figure 3A). Alterations in Wnt-pathway molecular expression and activity were then examined. AFP downregulated Axin 1 and pGSK3 $\beta$ and upregulated active $\beta$-catenin, TCF1/TCF7, and c-Myc in APGC cells and derived xenografts, indicating AFP-mediated Wnt-signaling activation (Figure 3B-D). Consistently, AFP promoted $\beta$-catenin-mediated TCF transcriptional activity, marked by an increased TOPflash:FOPflash ratio (Figure 3E). As such, AFP-mediated GC growth and progression might have been due to canonical Wnt-signaling activation.

\section{Wnt-signaling blockade reduced AFP- mediated Wnt-pathway activation and malignancy in established APGC cells}

Given Wnt signaling as a candidate downstream pathway of AFP, Wnt-pathway roles in GC phenotypes were first validated by siRNA-mediated Axin 1 knockdown. Compared to controls, Axin 1 knockdown strengthened cell-proliferation, -invasion, and -migration abilities through activating Wnt pathways (marked by reduced pGSK3 $\beta$ and cascade activation of $\beta$-catenin, TCF1/TCF7, and c-Myc; Figure 4A-D) in GC cells. The same phenotypes of Axin 1 knockdown as AFP overexpression (Figures 1D and 3) support our assumption of Wnt signaling being responsible for AFP-mediated malignancy. More importantly, Wnt-pathway changes and malignant biological behaviors (including cell proliferation, invasion, and migration) induced by AFP overexpression (Figures 1D and 3) were impeded by Axin 1 overexpression (Figure 4E-H). Meanwhile, the Wnt-pathway inhibitor XAV939 effectively inhibited Wnt signaling (marked by enhanced pGSK $3 \beta$ and decreased active $\beta$-catenin, TCF1/ TCF7, and c-Myc) and repressed growth, invasion, and migration in established APGC cells (Figure 5A-C). Therefore, targeting Wnt signaling by Axin 1 rescue or pathway inhibitor repressed proliferation, invasion, and migration in established APGC cells, suggesting Wnt-signaling inhibitors as a promising strategy for APGC.

\section{Discussion}

Metastasis commonly occurs in livers and predominantly contributes to cancer-related deaths in GC. ${ }^{25,26}$ As such, seeking molecular mechanisms of cancer progression is essential to develop novel targets and improve prognosis for GC. Due to its high incidence of liver metastasis and poor survival, ${ }^{2,5}$ APGC is considered a representative metastatic subtype of GC and holds promise to give new insights into optimal GC management. Several studies have evidenced a tight association between elevated serous AFP and cancer aggression in APGC patients, yet whether AFP initiates malignant biological behaviors and involved mechanisms, or potential therapies in APGC, remains elusive.

AFP is an oncogenic glycoprotein that can be normally synthesized by yolk sac and fetal liver during gestation and in infants, whereas it often increases abnormally in adults with cancers represented by $\mathrm{HCC} .{ }^{2}$ Apart from the diagnostic and predictive value of AFP in $\mathrm{HCC}$, AFP-mediated aggressive $\mathrm{HCC}$ behaviors involving tumorigenesis and metastasis have been clarified in preclinical models, ${ }^{7,9-11}$ which were validated in our work using AFP-knockdown HepG2 cells 
A

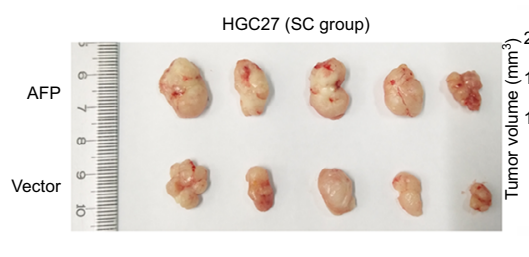

C

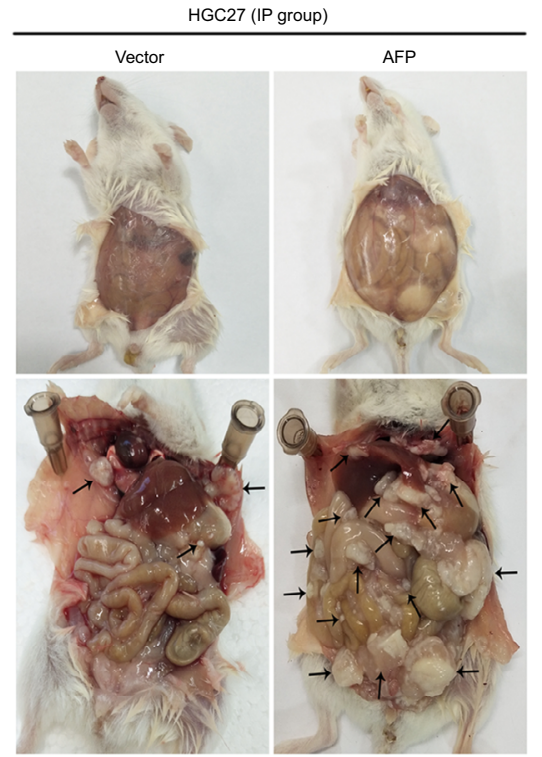

B

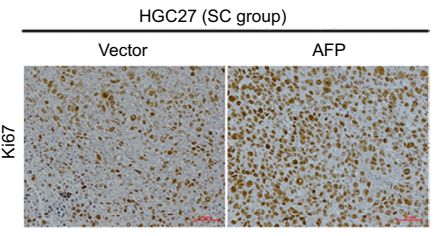

D

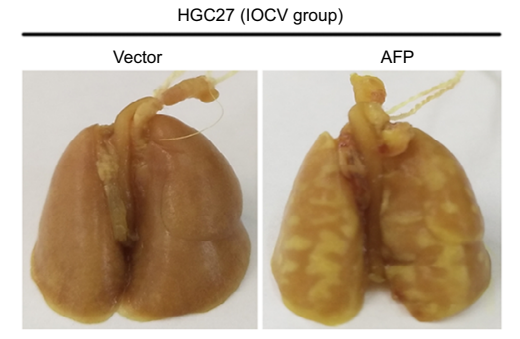

E

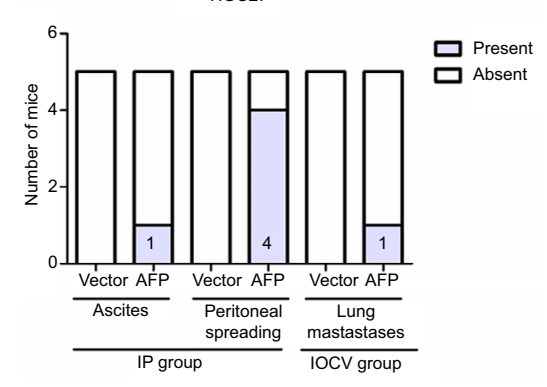

F

HGC27

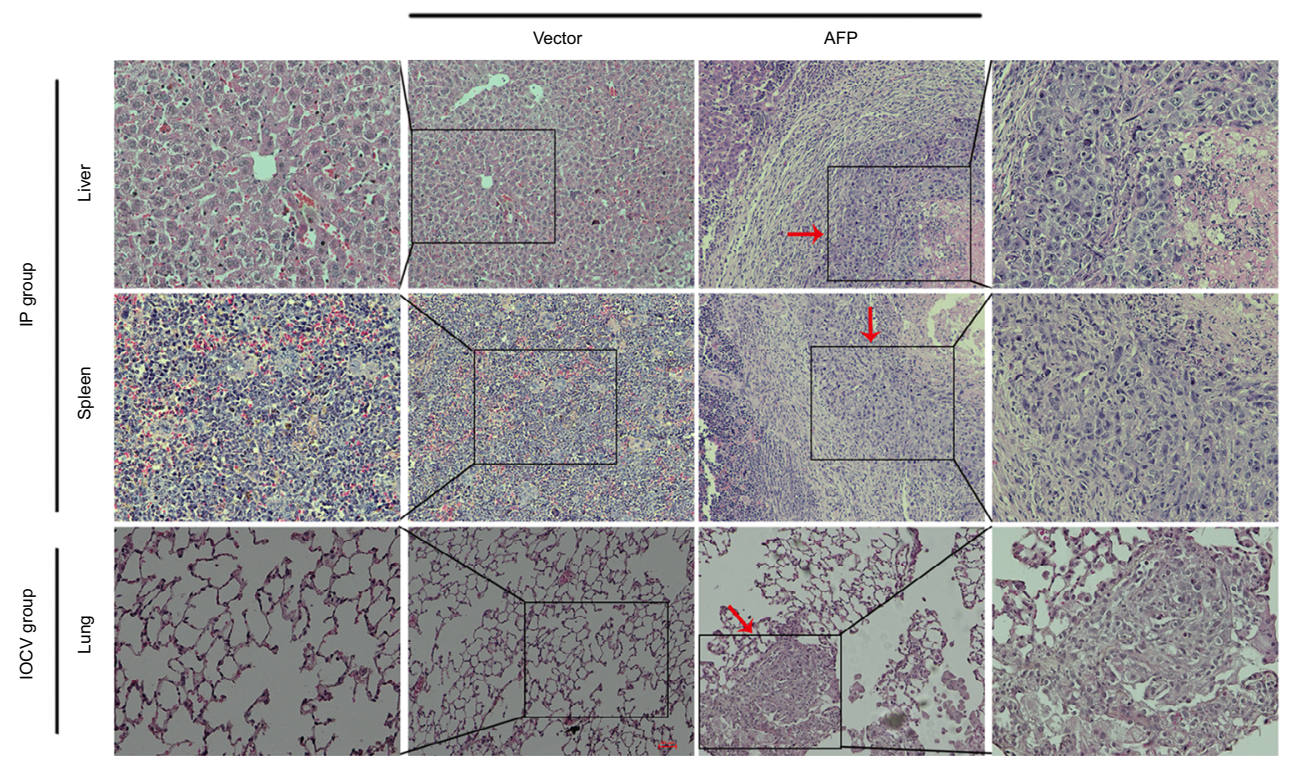

Figure 2 AFP promoted growth and metastasis in established APGC xenografts.

Notes: HGC27-AFP and HGC27-vector cells were subcutaneously injected into NOD/SCID mice (n=5/group). (A) Tumor volume was measured every 3 days after 2 weeks; tumor-growth curves shown. Data expressed as mean $\pm \mathrm{SD}$. ${ }^{* P}<0.05$ by repeated-measure ANOVA. (B) FFPE sections were stained with Ki67 for IHC analysis. NOD/SCID mice inoculated with HGC27-AFP and HGC27-vector cells by IP route and IOCV ( $n=5 /$ group). (C and D) Representative images of ascites and peritoneal dissemination in IP groups and lung metastasis in IOCV groups. Black arrows, disseminated tumor foci. (E) Comparisons of ascites, peritoneal dissemination, and distant metastasis capacity between mice bearing HGC27-AFP and HGC27-vector cells. (F) Representative H\&E-stained foci of peritoneal spreading in liver and spleen from IP groups and of distant metastasis in lung from IOCV groups. Red arrows, tumor foci.

Abbreviations: APGC, AFP-producing gastric cancer; FFPE, formalin-fixed, paraffin-embedded; IHC, immunohistochemistry; IOCV, injection of caudal vein; IP, intraperitoneal injection; NOD, nonobese diabetic; SC, subcutaneous; SCID, severe combined immunodeficiency. 
A

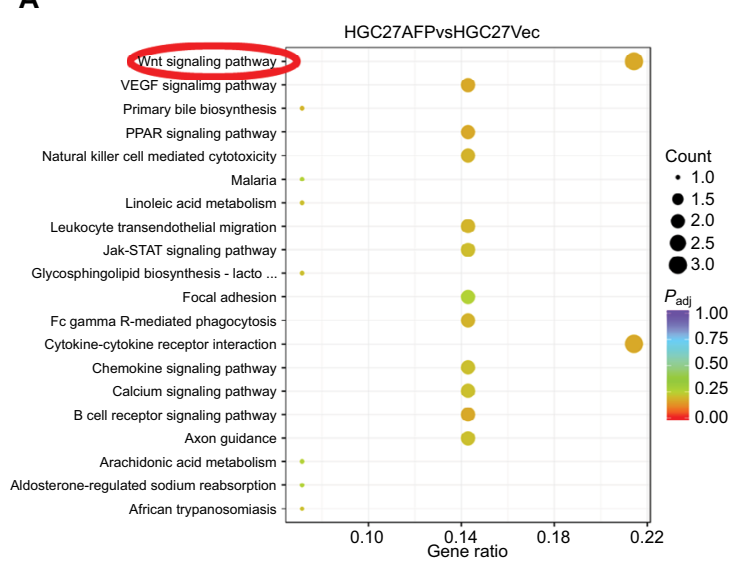

C

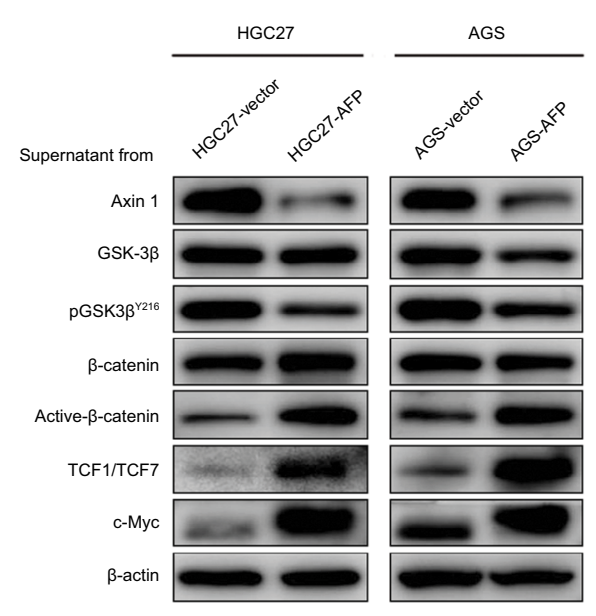

E
B

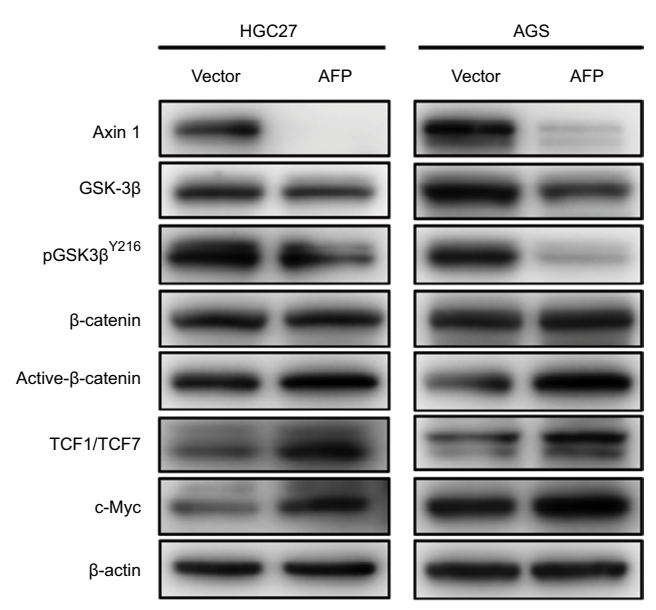

D

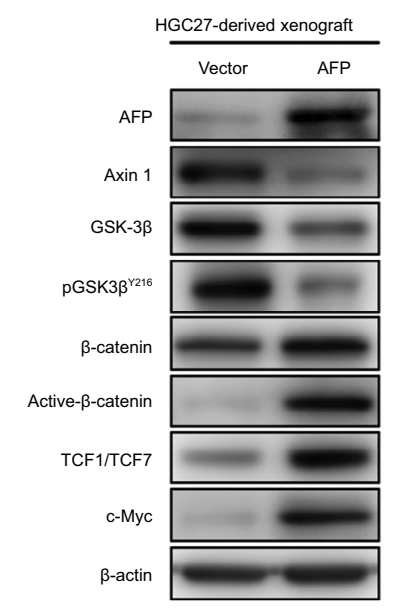

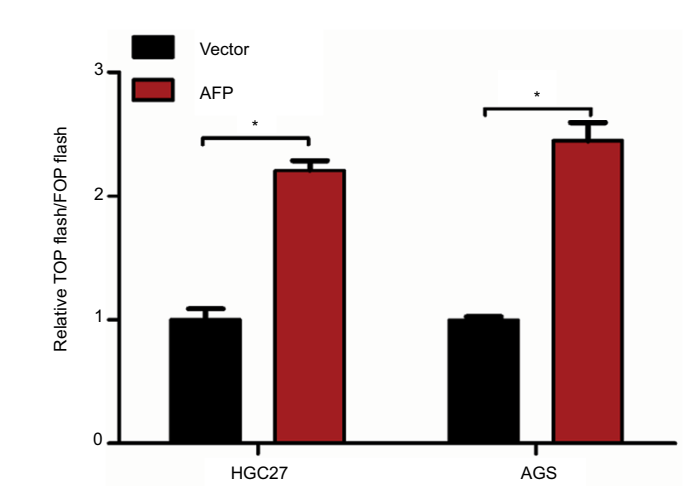

Figure 3 AFP-activated canonical Wnt signaling in established APGC preclinical models.

Notes: (A) The top 20 KEGG-identified candidate pathways enriched by a comparison of AFP-overexpressing HGC27 cells and the control. (B-D) Cellular, supernatant, and tissue proteins extracted from indicated groups were probed against a variety of Wnt-signaling-associated antibodies as indicated. (E) TOPflash and FOPflash plasmids were cotransfected with Renilla plasmids and subjected to dual-luciferase assays after 48 hours in AFP-overexpressing HGC27 and AGS cells and their controls. Reporter activity was normalized to Renilla luciferase activity. Data expressed as mean $\pm \mathrm{SD}$. $* P<0.05$ by ANOVA.

Abbreviations: APGC, AFP-producing gastric cancer; KEGG, Kyoto Encyclopedia of Genes and Genomes; $P_{\text {adj }}$ adjusted $P$-value.

(Figure S3). Tumorigenesis can also be initiated by AFP in cholangiocarcinoma and pancreatic cancer. ${ }^{27,28}$ Nonetheless, how AFP impacts GC, especially in excreted form, is largely unknown. Our data for the first time systematically showed
AFP-induced GC growth and metastasis in vitro and in vivo (Figures 1 and 2), consistent with previous sketchy observations of AFP-mediated in vitro invasion and in vivo growth in GC. ${ }^{12,13}$ APGC is frequently defined by an elevated level 
A

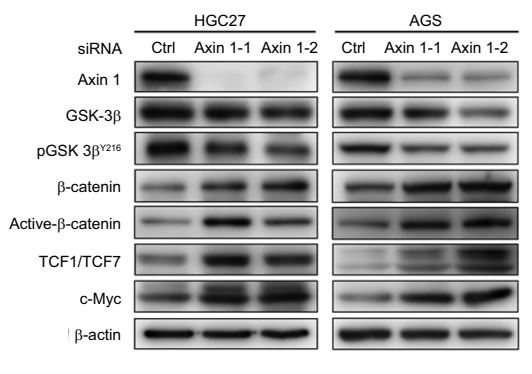

B

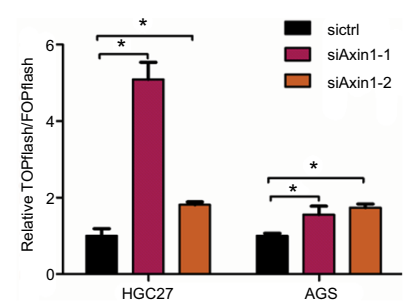

C

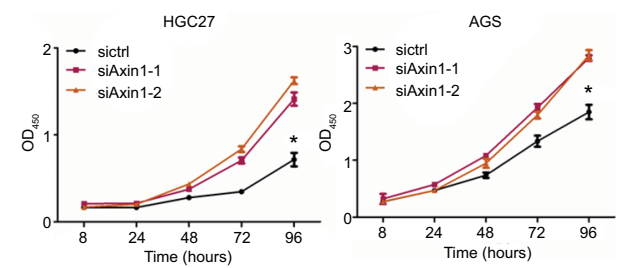

D
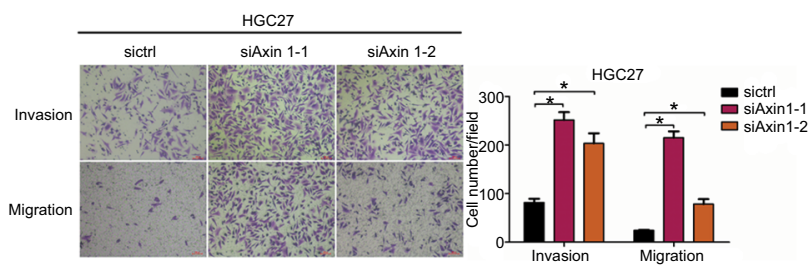

E

F
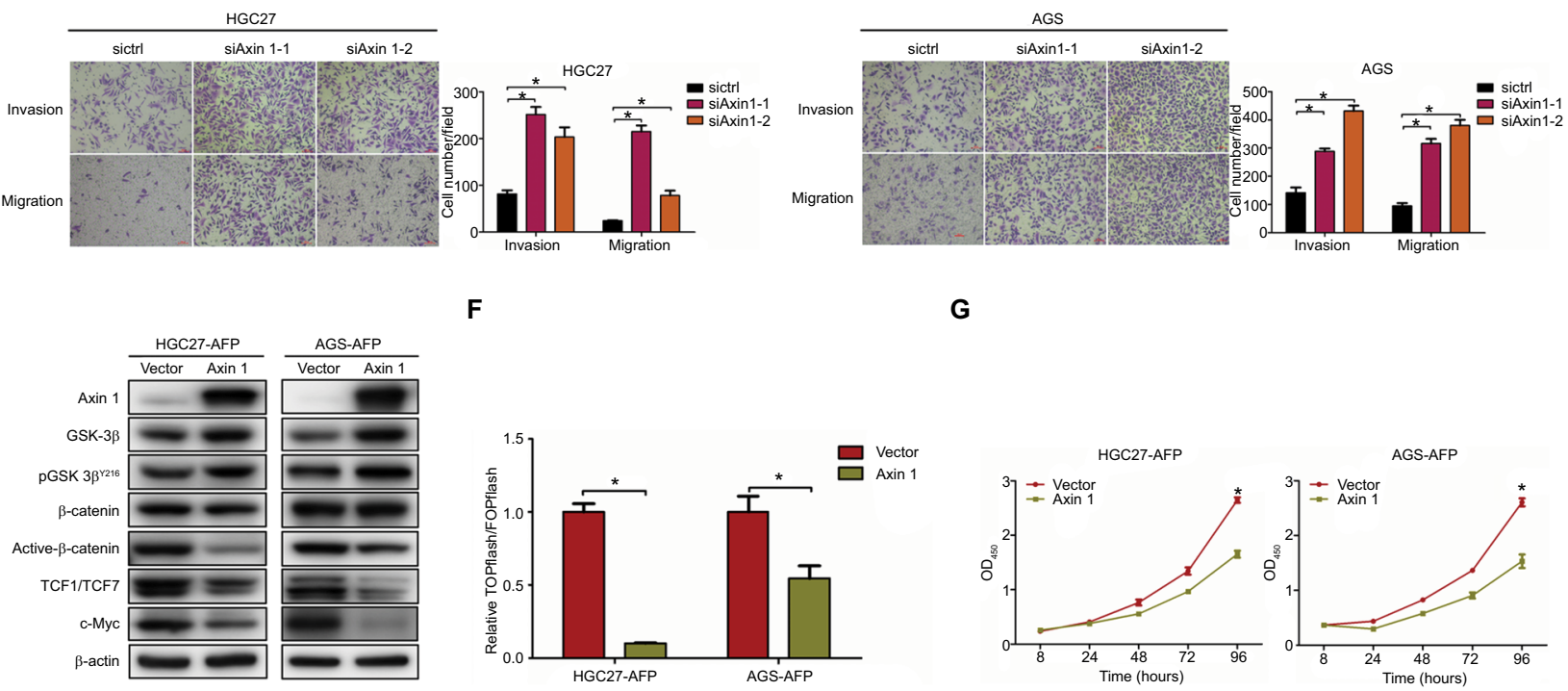

F

G
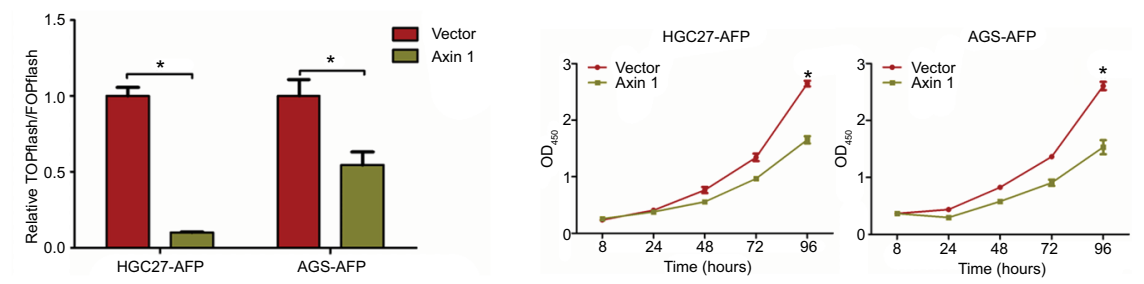

H
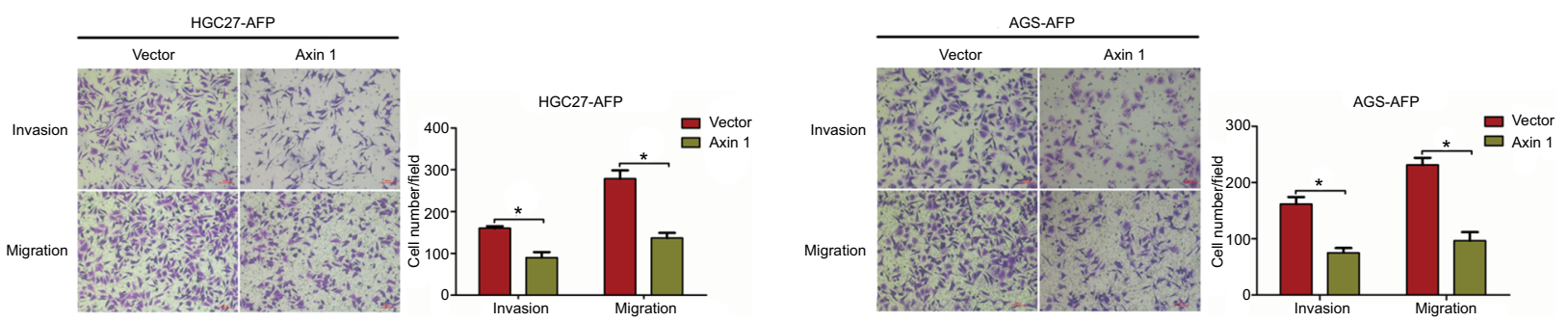

Figure 4 Axin I overexpression reduced AFP-mediated Wnt-pathway activation and malignancy in established APGC cells.

Notes: (A-D) After Axin I knockdown using siRNAs in GC cells and (E-H) Axin I overexpression in AFP-overexpressing GC cells for 48 hours, Wnt-signaling-involved protein-expression levels, $\beta$-catenin-mediated TCF transcriptional activity, and cell-proliferation, -invasion, and -migration abilities were determined by immunoblotting, dualluciferase, CCK8, and transwell assays, respectively. Data expressed as mean $\pm S D$. $* P<0.05$ by ANOVA.

Abbreviation: APGC, AFP-producing gastric cancer.

of serum AFP or positive immunohistochemical staining of AFP, also known as seropositive or "histopositive" APGC, respectively. ${ }^{2,4}$ As such, our work also initially harnessed AFP supernatant to better imitate APGC aggressiveness. As expected, AFP supernatant induced in vitro proliferation, invasion, and migration similar to AFP overexpression (Figure 1D and E). Notably, intracellular proteins of AFP were not detected by Western blots (Figure S1) despite its extracellular contents being robustly elevated (Figure $1 \mathrm{~B}$ and $\mathrm{C}$ ), indicating abundant AFP secretion in our AFP-overexpressing GC cells. AFP might function as a secretory protein to provoke oncogenic and metastatic features in GC, suggesting the therapeutic potential of targeting AFP and relevant signaling for APGC (Figure 6). To warrant the findings, AFP with bioactivity purified from supernatants should be employed for future investigations.

Of note, AFP prominently synthesized by fetal liver and highly expressed in hepatic stem cells/hepatoblasts serves as a diagnostic biomarker for HCC, and AFP production originates from hepatoid or enteroblastic differentiation of 
A

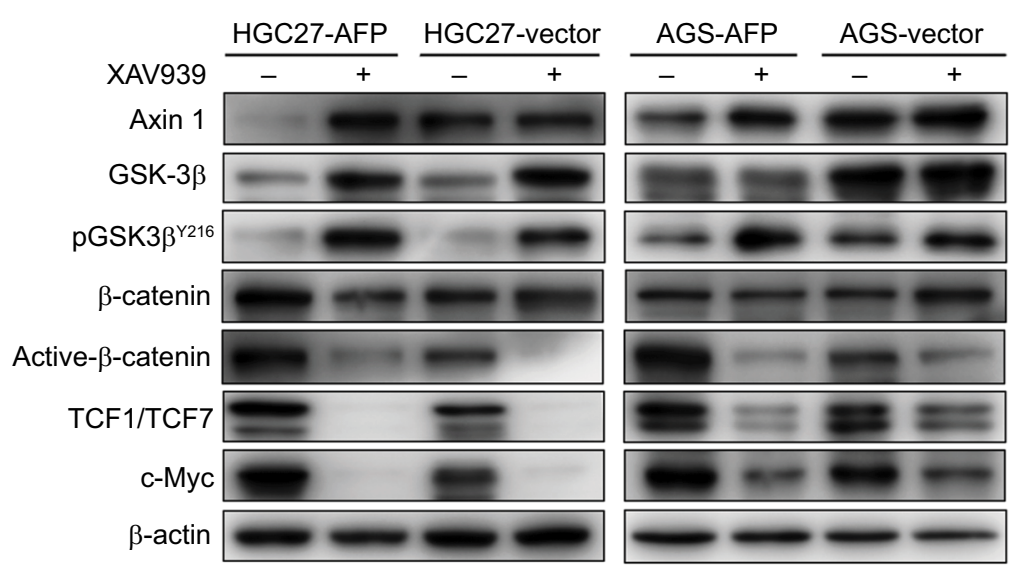

C
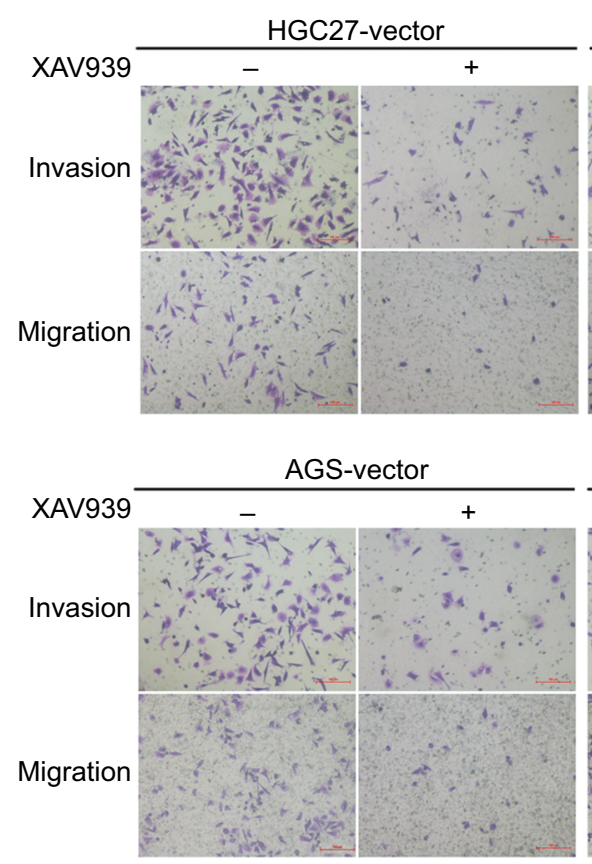

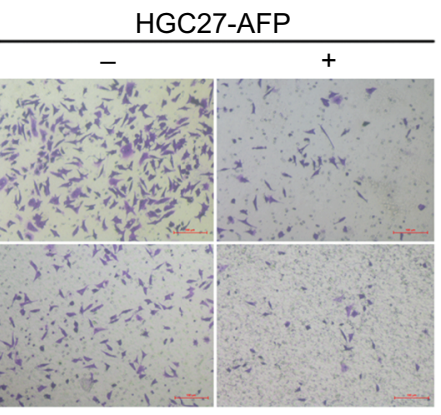

AGS-AFP

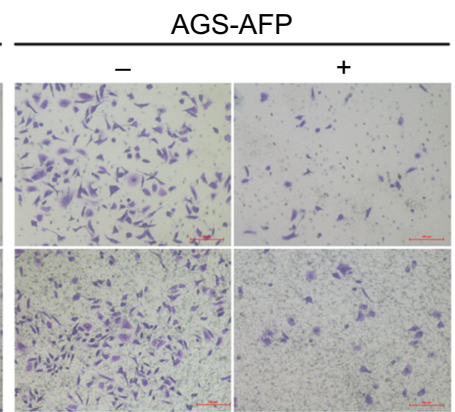

B
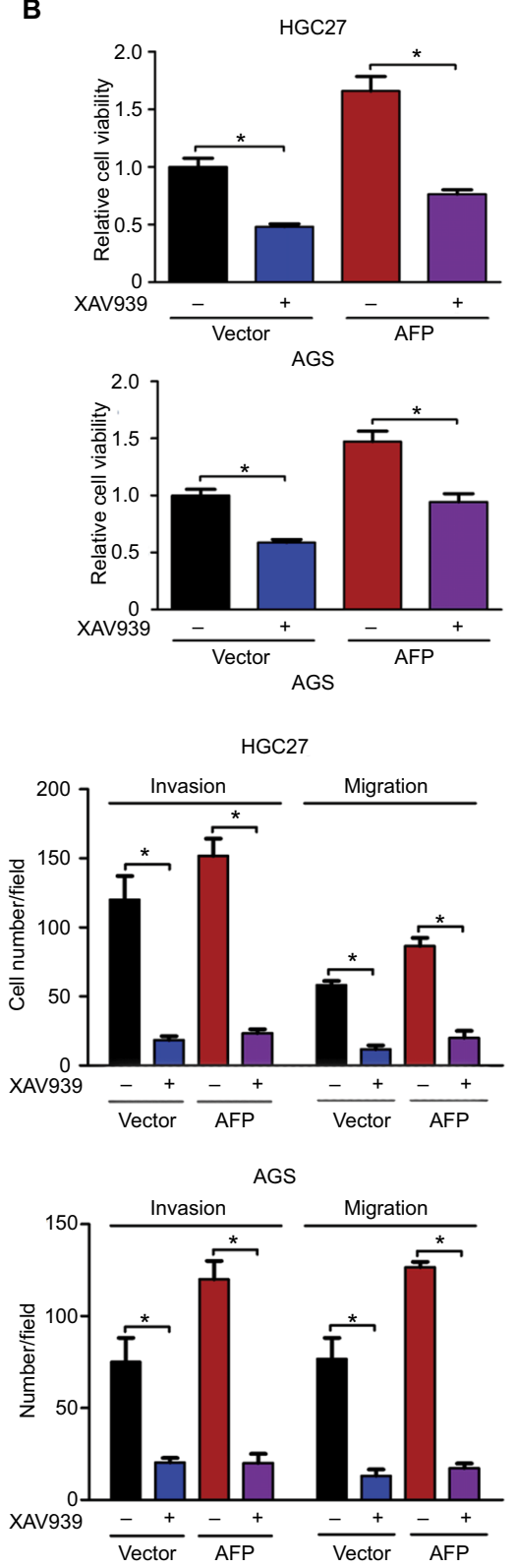

Figure 5 Wnt-pathway inhibitor reduced AFP-mediated Wnt-pathway activation and malignancy in established APGC cells.

Notes: AFP-overexpressing GC cells and their controls were treated in the absence or presence of Wnt-pathway inhibitor XAV939 (50 $\mu$ M) for 48 hours. (A) Immunoblotting was carried out for Wnt axis-associated proteins and (B and $\mathbf{C}$ ) proliferation and invasion/migration abilities were measured by CCK8 and Transwell assays with/without Matrigel, respectively. Data expressed as mean $\pm S D$. $* P<0.05$ by ANOVA.

Abbreviation: APGC, AFP-producing gastric cancer.

GC cells, ${ }^{2,29}$ indicating AFP's hepatotropic properties. With no liver metastasis observed in this study (data not shown), AFP-mediated in vivo progression needs further assessment using optimized liver-metastasis models, such as in vivo imaging systems. ${ }^{25}$ Despite AFP-promoted progression presented in vitro and in vivo, lung metastasis barely occurred in one in five mice. Therefore, optimized models are required to warrant a vital role of AFP in APGC aggression, such as using GC cell lines with higher metastasis tendencies, in vivo imaging systems to monitor metastasis formation in real time, survival experiments to assess long-term effects, AFP-overexpressed transgenic mice, and patient-derived xenografts of APGC compared with non-APGC.

Candidate molecules and signaling pathways of AFPassociated malignant phenotypes are eagerly awaited. The PI3K-Akt cascade and its regulated proteins have been reported to account for AFP-induced tumorigenesis and metastasis in AFP-producing HCC. ${ }^{7-10}$ However, this pathway 


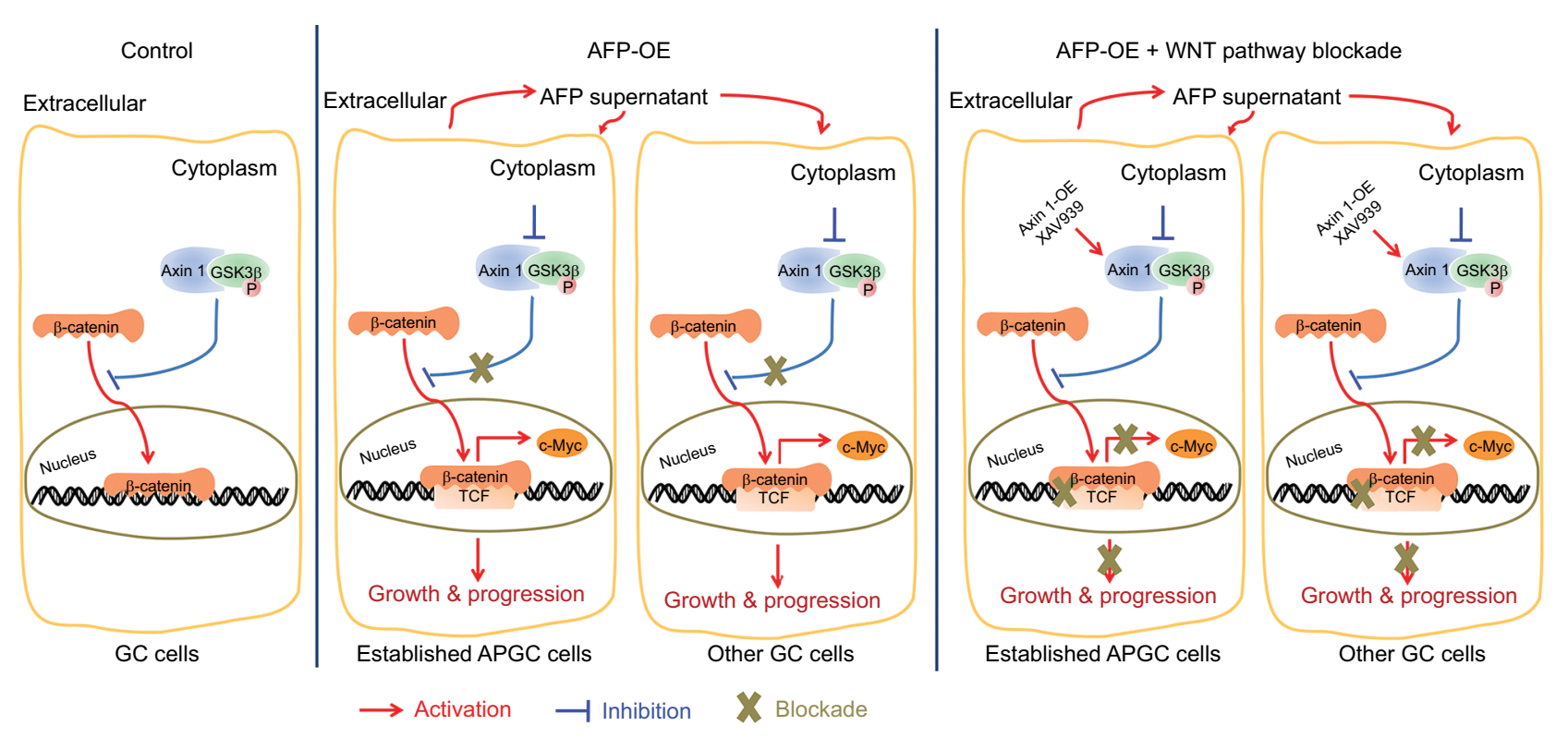

Figure 6 Representation of targeting Wnt pathways responsible for AFP-associated malignancy in APGC.

Notes: Compared to the control, AFP overexpression promoted AFP-supernatant secretion, which activated canonical Wnt signaling (marked by inactive destruction complex including GSK3 $\beta$ dephosphorylation/inactivation, and reduced Axin I, increased active $\beta$-catenin, augmented TCF transcriptional activity and upregulated target gene c-MYC) to enhance growth and metastasis in GC. Wnt-pathway blockade by Axin I overexpression or small-molecule inhibitor XAV939 (Axin I stabilizer) impeded AFP-mediated malignancy in AFP-overexpressing GC, indicating therapeutic potentials of targeting Wnt pathways against APGC.

Abbreviation: APGC, AFP-producing gastric cancer.

might be dispensable for AFP-driven malignancy in APGC denoted by no changes in PI3K-Akt signaling molecules after AFP overexpression in GC cells (Figure S2). This discrepancy could result from the heterogeneity among different cancer types. On the other hand, high expression of angiogenic molecules, including VEGF and c-Met, have been reported in APGC compared to common GC. ${ }^{19-21}$ AFP blockade with genetic knockdown or specific antibodies consistently reduce VEGF expression and exert anticancer efficacy in preclinical AFP-producing HCC and APGC. . $^{13,30}$ These data suggest targeting angiogenesis as a promising strategy to treat APGC, whereas AFP overexpression failed to increase VEGF expressions in our GC cells (Figure S2). Due to a close link between angiogenic factor functions and the tumor microenvironment, ${ }^{31}$ antiangiogenic strategies, such as the clinically approved apatinib and ramucirumab, ${ }^{1,32}$ against APGC still merit further in vivo evaluation.

Apart from angiogenic pathways, CSCs involved in tumor initiation, metastasis, and recurrence ${ }^{33,34}$ emerge as another compelling target for AFP-producing tumors. Resembling AFP's role in normal hepatic stem cells and hepatoblasts, ${ }^{29} \mathrm{AFP}$ renders CSC-like phenotypes in HCC, cholangiocarcinoma, and pancreatic cancer., ${ }^{7,27,28}$ Thereby, stem-cell biomarkers enriched in AFP-producing cancers and involved in AFP-driven malignancy have therapeutic potential, but remain far from clear. Of interest, Wnt signaling, a typical CSC-related pathway to endowment of GC-growth advantages and progression tendencies, ${ }^{35-37}$ may be practicable for APGC. Hepatic stem-cell-like HCC defined by $\mathrm{EPCAM}^{+} \mathrm{AFP}^{+} \mathrm{HCC}$ presents Wnt-signaling activation, suggesting that Wnt-signaling inhibitors might be able to eradicate this HCC subtype. ${ }^{29}$ Previous bioinformatic and IHC data uncovered Wnt-pathway activation in tissue of AFP-producing cancers, including APGC, represented by overexpressing GPC $3 .{ }^{15,17,38,39}$ In parallel with inactivated Wnt pathways in AFP-knockdown liver cancer cells (Figure S3A), canonical Wnt pathways screened by RNA-sequencing were activated in established APGC preclinical models, which contributed to AFP-mediated growth and progression (Figures 3-5). As such, Wnt-signaling blockade holds promises to benefit patients with APGC. Our data show anticancer efficacy and progression inhibition achieved by targeting the Wnt cascade with Axin 1 overexpression or pathway inhibitors in established APGC cells (Figures 4E-H and 5). More inhibitors targeting Wnt signaling are being subjected to phase I clinical trials on efficacy and safety in a series of cancers, such as CGX1321, Foxy5, SM08502, ETC1922159, DKN01, niclosamide, and resveratrol. Strikingly, effectiveness and tolerability of the anti-GPC3 antibody ERY974 are also being assessed in GPC3-positive advanced GC. Based on 
GPC3's diagnostic and predictive values in AFP-producing cancers $^{17,38,40}$ and good responses of our established APGC cells to targeting Wnt pathways (Figures 4E-H and 5), whether APGC patients are a more specific population than common advanced GC for these Wnt-related blockades, the GPC3 antibody in particular, is worthy of future studies.

Understanding how AFP acts on cancer cells and which molecules AFP interacts with to trigger its oncogenic effects are of great significance. For one thing, AFP expression is regulated by transcription factors, including transcription activators (like the hepatocyte nuclear factors $\mathrm{C} / \mathrm{EBP}$ and $\mathrm{NF} \kappa \mathrm{B}),{ }^{41,42}$ transcription repressors (like ATBF1), ${ }^{43,44}$ and transcription pioneers (like FOXA1). ${ }^{45}$ For another, multiple forms of AFP stemming from abundant posttranscriptional and posttranslational modifications induce oncogenic phenotypes via different protein-protein interactions. Firstly, cytoplasmic AFP colocalizes and interacts with caspase 3/XIAP, RAR, and PTEN to regulate apoptotic pathways, GADD153 expression/Fn14 transcription, and PI3K-Akt pathways, followed by activated CXCR4. ${ }^{46,47}$ Secondly, secreted AFP binds to AFP receptors expressed on plasmid membranes to promote tumor growth through $\mathrm{cAMP} / \mathrm{PKA} /$ intracellular calcium and the PI3K-Akt-Src axis. ${ }^{48,49}$ Intriguingly, AFP receptors can also be detected in sera to screen breast cancer at an early stage. ${ }^{50}$ Beyond AFP receptors, secreted AFP binds to CCR 5 chemokine receptors on macrophages, ${ }^{51}$ which indicates the presence of an AFP-associated tumorimmune microenvironment. Thirdly, the carboxyterminal third domain of AFP, containing a large fragment of amino acids and behaving similar to full-length AFP, can interact with various receptors, including scavenger receptors, scavenger-associated receptors, and cell-cycle proteins. ${ }^{52,53}$ Finally, AFP-L3 (a core-fucosylated AFP) in sera of prognostic and diagnostic value in $\mathrm{HCC}^{54-57}$ is lectin-bound. Despite the Wnt cascade being presented as a downstream AFP pathway in our work, whether AFP interacts with molecules involving Wnt signaling like Axin 1 remains obscure, and how AFP transmits oncogenic signals to Wnt pathways merits further investigations.

\section{Conclusion}

AFP overexpression and derived supernatant potentiated tumor growth and metastasis partially through Wnt-signaling activation in GC preclinical models. Wnt-signaling blockade had anticancer efficacy in established APGC cells, shedding light on the therapeutic potential of targeting Wnt pathways for APGC and providing novel choices to treat metastasis in $\mathrm{GC}$.

\section{Data sharing statement}

No genomic data were deposited in public repositories, due to privacy protection. Please contact the first authors Dongshao Chen (chendongshao@bjmu.edu.cn) and Xiaoting Lin(1411110421@bjmu.edu.cn) via email if you need them.

\section{Acknowledgments}

The authors thank Professor Youyong Lv (Peking University Cancer Hospital and Institute) for kindly providing the AGS cell line. This work was supported by funding from the National Key Research and Development Program of China (2017YFC1308900), Beijing Municipal Administration of Hospital Clinical Medicine Development of Special Funding Support (ZYLX201701), and Beijing Natural Science Foundation (7161002).

\section{Disclosure}

The authors report no conflicts of interest in this work.

\section{References}

1. Van Cutsem E, Sagaert X, Topal B, Haustermans K, Prenen H. Gastric cancer. Lancet. 2016;388(10060):2654-2664.

2. Sun W, Liu Y, Shou D, et al. AFP (alpha fetoprotein): who are you in gastrology? Cancer Lett. 2015;357(1):43-46.

3. Lin HJ, Hsieh YH, Fang WL, Huang KH, Li AF. Clinical manifestations in patients with alpha-fetoprotein-producing gastric cancer. Curr Oncol. 2014;21(3):394-399.

4. Wang YK, Shen L, Jiao X, Zhang XT. Predictive and prognostic value of serum AFP level and its dynamic changes in advanced gastric cancer patients with elevated serum AFP. World J Gastroenterol. 2018;24(2):266-273.

5. Hirajima S, Komatsu S, Ichikawa D. Liver metastasis is the only independent prognostic factor in AFP-producing gastric cancer. World $J$ Gastroenterol. 2013;19(36):6055-6061.

6. Liu X, Cheng Y, Sheng W, et al. Clinicopathologic features and prognostic factors in alpha-fetoprotein-producing gastric cancers: analysis of 104 cases. J Surg Oncol. 2010;102(3):249-255.

7. Zhu M, Li W, Lu Y. HBx drives alpha fetoprotein expression to promote initiation of liver cancer stem cells through activating PI3K/Akt signal pathway. Nanoscale. 2017;140(6):1346-1355.

8. Li M, Li H, Li C, et al. Alpha-fetoprotein: a new member of intracellular signal molecules in regulation of the PI3K/Akt signaling in human hepatoma cell lines. Int $J$ Cancer. 2011;128(3):524-532.

9. Gao R, Cai C, Gan J, et al. miR-1236 down-regulates alpha-fetoprotein, thus causing PTEN accumulation, which inhibits the PI3K/Akt pathway and malignant phenotype in hepatoma cells. Oncotarget. 2015;6(8):6014-6028.

10. Lu Y, Zhu M, Li W, et al. Alpha fetoprotein plays a critical role in promoting metastasis of hepatocellular carcinoma cells. J Cell Mol Med. 2016;20(3):549-558.

11. Yang X, Zhang Y, Zhang L, Zhang L, Mao J. Silencing alpha-fetoprotein expression induces growth arrest and apoptosis in human hepatocellular cancer cell. Cancer Lett. 2008;271(2):281-293.

12. Lu S, MaY, Sun T, Ren R, Zhang X, Ma W. Expression of $\alpha$-fetoprotein in gastric cancer AGS cells contributes to invasion and metastasis by influencing anoikis sensitivity. Oncol Rep. 2016;35(5):2984-2990.

13. Takahashi Y, Ohta T, Mai M. Angiogenesis of AFP producing gastric carcinoma: correlation with frequent liver metastasis and its inhibition by anti-AFP antibody. Oncol Rep. 2004;11(4):809-813. 
14. Yokoo H, Kondo T, Fujii K, Yamada T, Todo S, Hirohashi S. Proteomic signature corresponding to alpha fetoprotein expression in liver cancer cells. Hepatology. 2004;40(3):609-617.

15. Saito S, Ojima H, Ichikawa H, Hirohashi S, Kondo T. Molecular background of alpha-fetoprotein in liver cancer cells as revealed by global RNA expression analysis. Cancer Sci. 2008;99(12):2402-2409.

16. He L, Ye F, Qu L, et al. Protein profiling of alpha-fetoprotein producing gastric adenocarcinoma. Oncotarget. 2016;7(19):28448-28459.

17. Murakami T, Yao T, Mitomi H, et al. Clinicopathologic and immunohistochemical characteristics of gastric adenocarcinoma with enteroblastic differentiation: a study of 29 cases. Gastric Cancer. 2016;19(2): 498-507.

18. Osada M, Aishima S, Hirahashi M, et al. Combination of hepatocellular markers is useful for prognostication in gastric hepatoid adenocarcinoma. Hum Pathol. 2014;45(6):1243-1250.

19. Kamei S, Kono K, Amemiya H, et al. Evaluation of VEGF and VEGFC expression in gastric cancer cells producing alpha-fetoprotein. $J$ Gastroenterol. 2003;38(6):540-547.

20. Amemiya $\mathrm{H}$, Kono K, Takahashi A, et al. c-Met expression in a gastric cancer cell line producing alpha-fetoprotein. Surg Today. 2004;34(2):115-122.

21. Amemiya H, Kono K, Mori Y, et al. High frequency of c-Met expression in gastric cancers producing alpha-fetoprotein. Oncology. 2000;59(2):145-151.

22. Zhang Q, Zhu M, Cheng $\mathrm{W}$, et al. Downregulation of $425 \mathrm{G}>\mathrm{A}$ variant of calcium-binding protein S100A14 associated with poor differentiation and prognosis in gastric cancer. J Cancer Res Clin Oncol. 2015;141(4): 691-703.

23. Pan Y, Huang J, Xing R, et al. Metallothionein $2 \mathrm{~A}$ inhibits NF- $\mathrm{KB}$ pathway activation and predicts clinical outcome segregated with TNM stage in gastric cancer patients following radical resection. JTransl Med. 2013;11(1):173.

24. Chen D, Lin X, Zhang C, et al. Dual PI3K/mTOR inhibitor BEZ235 as a promising therapeutic strategy against paclitaxel-resistant gastric cancer via targeting PI3K/Akt/mTOR pathway. Cell Death Dis. 2018;9(2):123.

25. Zhang Y, Zhang Q, Zhang M, et al. DC-SIGNR by influencing the lncRNA HNRNPKP2 upregulates the expression of CXCR4 in gastric cancer liver metastasis. Mol Cancer. 2017;16(1):78.

26. Gong J, Tan G, Sheng N, You W, Wang Z. Targeted treatment of liver metastasis from gastric cancer using specific binding peptide. Am J Transl Res. 2016;8(5):1945-1956.

27. Sasaki N, Ishii T, Kamimura R, et al. Alpha-fetoprotein-producing pancreatic cancer cells possess cancer stem cell characteristics. Cancer Lett. 2011;308(2):152-161.

28. Ishii T, Yasuchika K, Suemori H, Nakatsuji N, Ikai I, Uemoto S. Alphafetoprotein producing cells act as cancer progenitor cells in human cholangiocarcinoma. Cancer Lett. 2010;294(1):25-34.

29. Yamashita T, Forgues M, Wang W, et al. EpCAM and alpha-fetoprotein expression defines novel prognostic subtypes of hepatocellular carcinoma. Cancer Res. 2008;68(5):1451-1461.

30. Meng W, Li X, Bai Z, et al. Silencing alpha-fetoprotein inhibits VEGF and MMP-2/9 production in human hepatocellular carcinoma cell. PLoS One. 2014;9(2):e90660.

31. Mittal K, Ebos J, Rini B. Angiogenesis and the tumor microenvironment: vascular endothelial growth factor and beyond. Semin Oncol. 2014;41(2):235-251.

32. Li J, Qin S, Xu J, et al. Randomized, double-blind, placebo-controlled phase III trial of apatinib in patients with chemotherapy-refractory advanced or metastatic adenocarcinoma of the stomach or gastroesophageal junction. J Clin Oncol. 2016;34(13):1448-1454.

33. Pützer BM, Solanki M, Herchenröder O. Advances in cancer stem cell targeting: how to strike the evil at its root. Adv Drug Deliv Rev. 2017;120:89-107.

34. Adorno-Cruz V, Kibria G, Liu X, et al. Cancer stem cells: targeting the roots of cancer, seeds of metastasis, and sources of therapy resistance. Cancer Res. 2015;75(6):924-929.

35. Mao J, Liang Z, Zhang B. Ubr2 enriched in $\mathrm{p} 53$ deficient mouse bone marrow mesenchymal stem cell-exosome promoted gastric cancer progression via Wnt/beta-catenin pathway. 2017;35(11):2267-2279.
36. Santos JC, Carrasco-Garcia E, Garcia-Puga M, et al. SOX9 elevation acts with canonical Wnt signaling to drive gastric cancer progression. Cancer Res. 2016;76(22):6735-6746.

37. Mao J, Fan S, Ma W, et al. Roles of Wnt/ $/$-catenin signaling in the gastric cancer stem cells proliferation and salinomycin treatment. Cell Death Dis. 2014;5(1):e1039.

38. Hishinuma M, Ohashi KI, Yamauchi N, et al. Hepatocellular oncofetal protein, glypican 3 is a sensitive marker for alpha-fetoprotein-producing gastric carcinoma. Histopathology. 2006;49(5):479-486.

39. Gao W, Kim H, Feng M, et al. Inactivation of Wnt signaling by a human antibody that recognizes the heparan sulfate chains of glypican-3 for liver cancer therapy. Hepatology. 2014;60(2):576-587.

40. Zhou F, Shang W, Yu X, Tian J. Glypican-3: a promising biomarker for hepatocellular carcinoma diagnosis and treatment. Med Res Rev. 2018;38(2):741-767.

41. Li HM, Ikeda H, Nakabayashi H, Nishi S, Sakai M. Identification of CCAAT enhancer binding protein alpha binding sites on the human alpha-fetoprotein gene. Gene. 2007;389(2):128-135.

42. Ye G, Sun G, Cheng Z, et al. P55PIK regulates alpha-fetoprotein expression through the NF- $\mathrm{KB}$ signaling pathway. Life Sci. 2017;191:104-110.

43. Kataoka H, Miura $Y$, Joh T, et al. Alpha-fetoprotein producing gastric cancer lacks transcription factor ATBF1. Oncogene. 2001;20(7): 869-873.

44. Zhang Z, Yamashita H, Toyama T, et al. ATBF1-a messenger RNA expression is correlated with better prognosis in breast cancer. Clin Cancer Res. 2005;11(1):193-198.

45. Yu W, Qiao Y, Tang X, et al. Tumor suppressor long non-coding RNA, MT1DP is negatively regulated by YAP and runx 2 to inhibit FOXA1 in liver cancer cells. Cell Signal. 2014;26(12):2961-2968.

46. Mizejewski GJ. Nonsecreted cytoplasmic alpha-fetoprotein: a newly discovered role in intracellular signaling and regulation. An update and commentary. Tumor Biol. 2015;36(12):9857-9864.

47. Dudich E, Semenkova L, Dudich I, Denesyuk A, Tatulov E, Korpela T. Alpha-fetoprotein antagonizes X-linked inhibitor of apoptosis protein anticaspase activity and disrupts XIAP-caspase interaction. FEBS $J$. 2006;273(16):3837-3849.

48. Zhu M, Guo J, Li W, et al. HBx induced AFP receptor expressed to activate PI3K/Akt signal to promote expression of SRC in liver cells and hepatoma cells. BMC Cancer. 2015;15(1):362.

49. Li MS, Li PF, He SP, Du GG, Li G. The promoting molecular mechanism of alpha-fetoprotein on the growth of human hepatoma Bel7402 cell line. World J Gastroenterol. 2002;8(3):469-475.

50. Moro R, Gulyaeva-Tcherkassova J, Stieber P. Increased alpha-fetoprotein receptor in the serum of patients with early-stage breast cancer. Curr Oncol. 2012;19(1):e1-8.

51. Zubkova E, Semenkova L, Dudich E, Dudich I, Parfyonova Y, Menshikov M. Alpha-fetoprotein contributes to THP-1 cell invasion and chemotaxis via protein kinase and Gi-protein-dependent pathways. Mol Cell Biochem. 2013;379(1-2):283-293.

52. Mizejewski GJ. The alpha-fetoprotein (AFP) third domain: a search for AFP interaction sites of cell cycle proteins. Tumor Biol. 2016;37(9):12697-12711.

53. Mizejewski GJ. The alpha-fetoprotein third domain receptor binding fragment: in search of scavenger and associated receptor targets. J Drug Target. 2015;23(6):538-551.

54. Choi J, Kim G-A, Han S, Lee W, Chun S, Lim Y-S. Longitudinal assessment of three serum biomarkers to detect very early stage hepatocellular carcinoma. Hepatology. In press 2018.

55. Ma H, Sun X, Chen L, et al. Multiplex immunochips for highaccuracy detection of AFP-L3\% based on surface-enhanced Raman scattering: implications for early liver cancer diagnosis. Anal Chem. 2017;89(17):8877-8883.

56. Li J, Gao T, Gu S, Zhi J, Yang J, Li G. An electrochemical biosensor for the assay of alpha-fetoprotein-L3 with practical applications. Biosens Bioelectron. 2017;87:352-357.

57. Yi CH, Weng HL, Zhou FG, et al. Elevated core-fucosylated IgG is a new marker for hepatitis B virus-related hepatocellular carcinoma. Oncoimmunology. 2015;4(12):e1011503. 


\section{Supplementary materials}

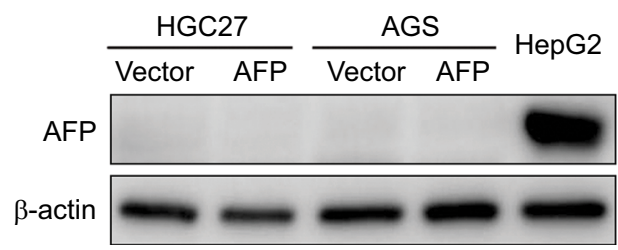

Figure SI Impact of AFP overexpression on AFP protein expression in gastric cancer cells.

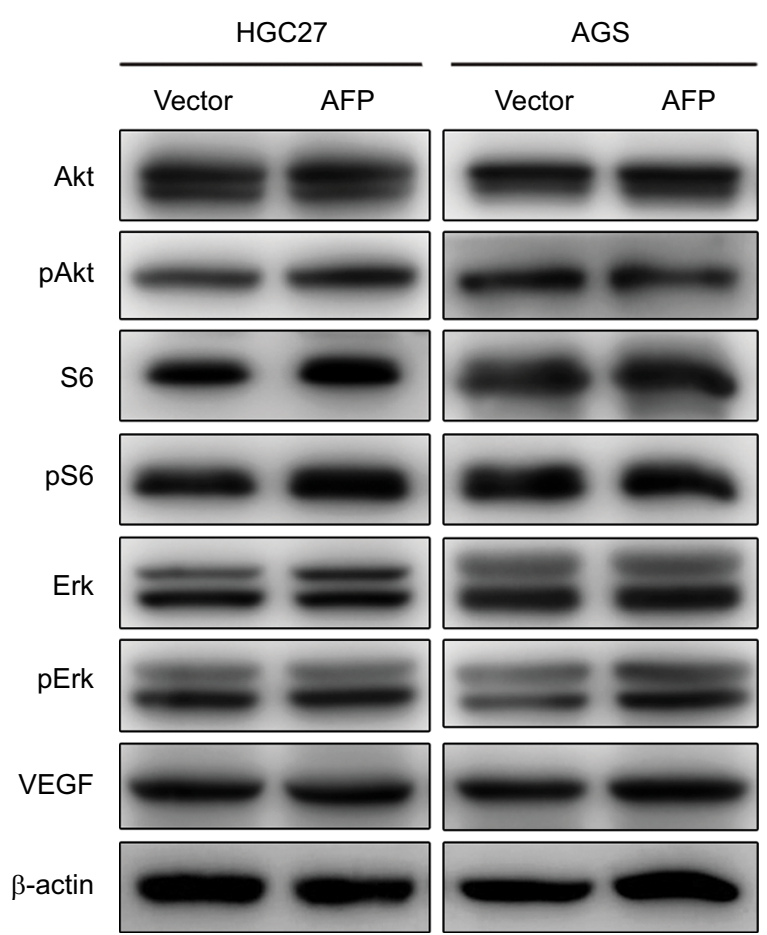

Figure S2 AFP overexpression had no effects on PI3K/Akt signaling or VEGF expression in gastric cancer cells.

Notes: Proteins extracted from AFP-overexpressing gastric cancer cells and their controls were probed against indicated antibodies. 
A

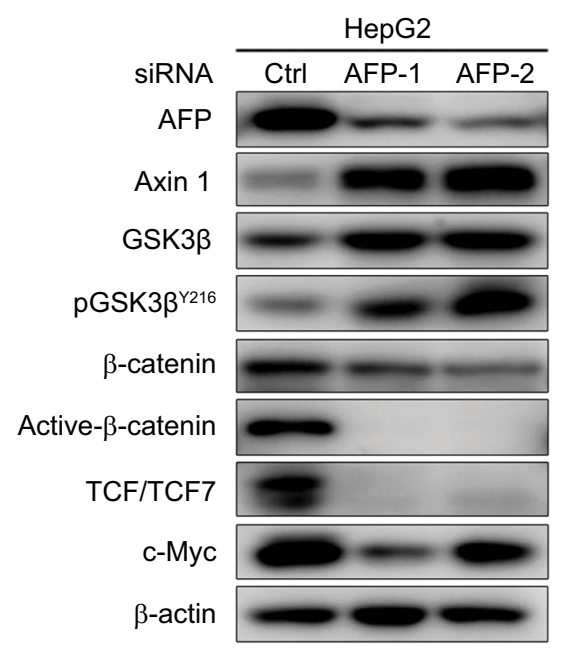

C

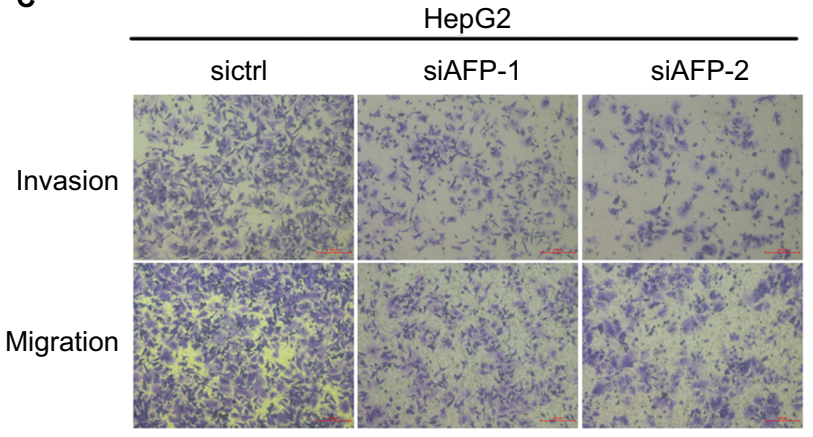

B

HepG2
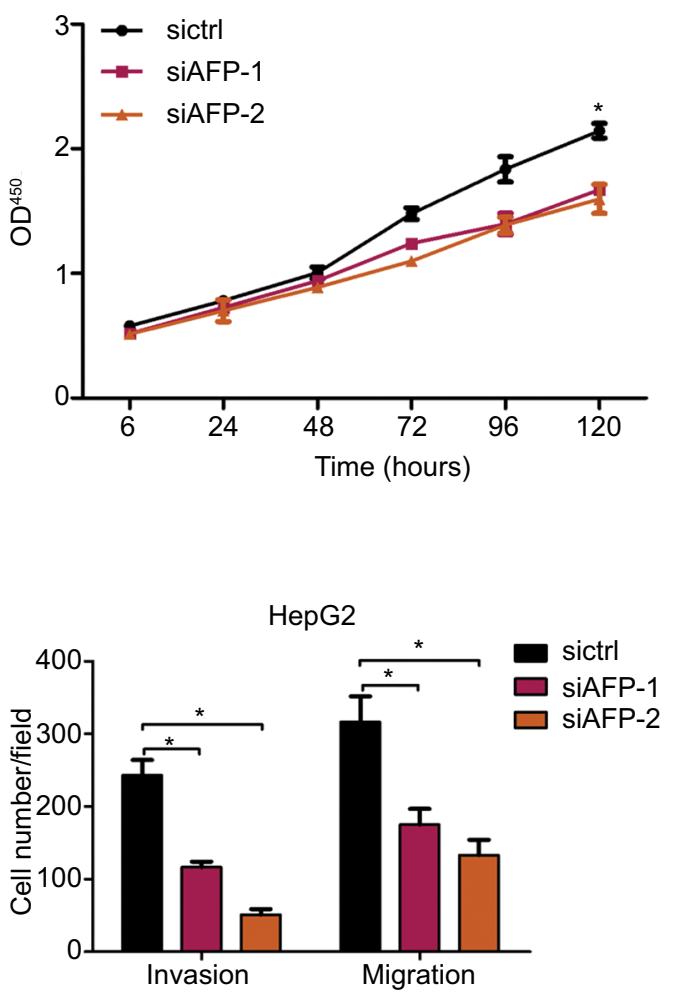

Figure S3 AFP knockdown inactivated the Wnt cascade and inhibited growth, invasion, and migration in HepG2 cells.

Notes: Proteins were probed against indicated antibodies involving Wnt signaling, and cell growth, invasion, and migration were compared in AFP-knockdown HepG2 cells and controls. Data expressed as mean $\pm \mathrm{SD}$. $* P<0.05$ by ANOVA analysis.

\section{Publish your work in this journal}

Cancer Management and Research is an international, peer-reviewed open access journal focusing on cancer research and the optimal use of preventative and integrated treatment interventions to achieve improved outcomes, enhanced survival and quality of life for the cancer patient. The manuscript management system is completely online and includes a very quick and fair peer-review system, which is all easy to use. Visit http://www.dovepress.com/testimonials.php to read real quotes from published authors. 